\title{
Clostridium oceanicum
}

National Cancer Institute

\section{Source}

National Cancer Institute. Clostridium oceanicum. NCI Thesaurus. Code C86286.

A species of anaerobic, Gram positive, rod shaped bacteria assigned to the phylum

Firmicutes. This species is indole negative, ferments cellobiose, glucose, mannose and

maltose but not ribose and does not reduce nitrate. C. oceanicum is considered nonpathogenic and has been isolated from marine sediments. 